\title{
Copula modelling of agitation-sedation rating of ICU patients: towards monitoring and alerting tools
}

\author{
$\underline{\text { A. Tursunalieva }}^{\mathrm{a}}{ }_{\oplus}$ and I. Hudson ${ }^{\mathrm{b}}{ }_{\odot}$ and J. G. Chase ${ }^{\mathrm{c}}{ }_{\odot}$ \\ ${ }^{a}$ School of Health Sciences, Swinburne University of Technology, Victoria, Australia, 3122 \\ ${ }^{\mathrm{b}}$ Department of Mathematical Sciences, Royal Melbourne Institute of Technology, Victoria 3000, Australia \\ ${ }^{\mathrm{c}}$ Department of Mechanical Engineering, University of Canterbury, Christchurch 8140, New Zealand \\ Email: atursunalieva@swin.edu.au
}

\begin{abstract}
Agitation-Sedation (A-S) cycling in critically ill intensive care unit (ICU) patients is damaging to health. Sedation quality is assessed by nurses and may suffer from subjectivity in their judgment and lead to sub-optimal sedation. Therefore, the use of quantitative modelling to enhance understanding of the A-S system is a key tool for optimising sedation management. Inadequate assessment of the agitation associated with clinical outcomes may lead to under or over-sedation and harm a patient's wellbeing.
\end{abstract}

Empirical distributions of the nurses' ratings of a patient's pain and/or agitation levels and the administered dose of sedative are often positively skewed and if the joint distribution is non-elliptical, then the high nurses' ratings of a patient's agitation levels may not correspond to the occurrences of patient's A-S profile with large infusion dose. Copulas measure nonlinear dependencies capturing the dependence between skewed distributions. Therefore, the aim is to use a copula-based dependence measure between the nurses' rating of patients' agitation level, and the automated sedation dose to identify patient-specific thresholds that separate the regions of mild, moderate, and severe agitation intensities. Delineating the occurrences with different agitation intensities allows us to establish the regions where nurses' rating has stronger or weaker correlation with the automated sedation dose.

This pilot study modelled agitation-sedation profiles of $\mathrm{t}$ wo $\mathrm{p}$ atients $\mathrm{c}$ ollected a $\mathrm{C}$ hristchurch Hospital, Christchurch School of Medicine and Health Sciences, NZ, from the pool of 37 patients. Classification of patients into poor and good trackers based on Wavelet Probability Bands (WPB). One of the patients is a poor tracker and the other patient is a good tracker. The best-fitting copula shows that the dependency structure between the nurses rating of a patients agitation level and the administered dose of sedative for both patients has an upper and lower tail. More specifically, a correlation between the nurses rating of a patients agitation severity and the administered dose of the sedative is the strongest when patients are expressing signs of a mild agitation, namely in the lower tail region (below the lower threshold for nurses' rating: 1.1 for poor tracker and 1.3 for good tracker) and weakest when patients are expressing signs of a severe agitation, namely in the upper tail region (above the upper threshold for nurses' rating: 2.6 for poor tracker and 6.1 for good tracker). The results show that for a good tracker, the nurses' rating of the patients' agitation levels has strong positive correlation with the administered dose of the sedative for low and mild agitation severity. For a poor tracker, the nurses' rating of the patients' agitation levels has strong positive correlation with the administered dose of the sedative only for low agitation severity. In addition, incorporating the tail dummy variables improved predictions of the nurses' rating by increasing the adjusted $R^{2}$ values by $28 \%$. Moreover, the percentage of lower and upper tail observations that are common with the lower and upper WPBs is higher for the poor tracker than the good tracker. However, the percentage of observations that are common to both the main region (the region associated with moderate agitation intensity) and within WPB is higher for the good tracker compared to the poor tracker.

In this paper we have accounted for non-linear relationships between the two variables, finding thresholds and regions of mismatch between the nurse's scores and sedation dose, thereby suggesting a possible way forward for an improved alerting system for over/under-sedation. Establishing the presence of tail dependence and patient-specific thresholds for areas with different agitation intensities has significant implications for the effective administration of sedatives. Better management of A-S states will allow clinicians to improve the efficacy of care and reduce healthcare costs.

Keywords: ICU, copula modelling, agitation-sedation assessment, nurses' rating, waverlet band 
A. Tursunalieva et al., Copula modelling of agitation-sedation rating ...

\section{INTRODUCTION}

Nurses play a major role in rating a patient's agitation levels. Assessing the severity of agitation is a challenging clinical problem as variability related to drug metabolism for each individual is often subjective. A multitude of previous studies suggests that the assessment accuracy of the sedation quality conducted by nurses tends to suffer from subjectivity and lead to sub-optimal sedation (Chase et al. (2004), Milane (1992)). For example, Barr et al. (2013) strongly recommend lighter than deeper levels of sedations. Moreover, Vincent et al. (2016) argue that sedation should be reviewed and adjusted regularly. Current agitation management methods rely on subjective agitation assessment and an appropriate sedation cycling in critically ill patients involves oscillations between states of agitation and over-sedation, which is detrimental to patient health and increases hospital length of stay (Rudge et al. (2005), Rudge et al. (2006a), Rudge et al. (2006b), Chase et al. (2004)).

The work of Rudge et al. (2006b) aimed to enhance feedback protocols for medical decision support systems and eventually automated sedation administration. A minimal differential equation model to predict or simulate each patients agitation-sedation status over time was presented in Rudge et al. (2006a) for 37 ICU patients and was shown to capture patient A-S dynamics. Current agitation management methods rely on subjective agitation assessment and an appropriate sedation input response from recorded at bedside agitation scales (Fraser and Riker (2001), Sessler et al. (2002)). The carers then select an appropriate infusion rate based upon their evaluation of these scales, their experience, and intuition (Kress et al. (2002)). This approach usually leads to largely continuous infusions which lack a bolus-focused approach, commonly resulting in over- or under-sedation. A more refined A-S model, which utilised regression with an Epanechnikov kernel was formulated by Rudge et al. (2006b). A Bayesian approach using densities and wavelet shrinkage methods was later suggested as a means to assess a previously derived deterministic, parametric A-S model (Kang et al. (2011), Kang et al. (2013)) and has successfully challenged the practice of sedating ICU patients using continuous infusions. Specifically, Kang et al. (2011) and Kang et al. (2013) have shown that wavelets provide a diagnostic and visualization tool to assess A-S models, and provided alternative numerical metrics of A-S control in assessing the validity of the earlier developed A-S deterministic dynamic models (see Table 3 in Kang et al. (2011)).

The current paper aims to build on earlier works of Tursunalieva et al. (2018) and Tursunalieva et al. (2019) to address the gap in the methodology by integrating non-elliptical dependence structure between nurses' rating of a patient's agitation level and the automated sedation dose, thereby accounting for non-linear relationships between the two variables. A similar approach was used by Boero et al. (2010) to capture the non-elliptical dependence between exchange rates. In an earlier work Tursunalieva et al. (2018) the tail thresholds were determined manually, whereas in Tursunalieva et al. (2019) the dynamic programming algorithm Bai and Perron (2003) was used to establish the upper tail threshold. In this paper, we identify both the lower and upper tail thresholds.

Copula (Nelsen (2006), Embrechts and McNeil (2003)) is a multivariate functional form for the joint distribution of random variables derived from pre-specified parametric marginal distributions of each random variable. The copula-based approach retains a parametric specification for the bivariate dependency but allows testing of several parametric structures to characterise the dependence, including one between skewed distributions. The empirical context in the current paper is a model of nurses' rating of agitation level concerning the automated simulated sedation dose. Copulas allow the strength of dependence to vary in different quantiles. The Kendall plot is used to determine the quantiles with significantly different strength of dependence. Establishing the regions with mild, moderate, and severe agitation intensities between nurses' rating, and the automated sedation dose allow us to identify occurrences where nurses are more likely to over and under-estimate the patient-specific agitation severity levels.

\section{METHODOLOGY}

\subsection{Data and statistical approach}

This study uses two intensive care unit patients' agitation-sedation profiles collected at Christchurch Hospital, School of Medicine and Health Sciences, NZ. Two variables were recorded for each patient: (1) the nurses' ratings of a patient's agitation level and (2) an automated sedation dose. Infusion data were recorded using an electronic drug infusion device for all admitted ICU patients during a nine-month observation period and required more than 24 hours of sedation. Infusion data containing less than 48 hours of continuous data, or data from patients whose sedation requirements were extreme, such as those with severe head injuries, were excluded. A total of $37 \mathrm{ICU}$ patients met these requirements and were enrolled in the oroginal study. 
The Kendall plot (K-plot) is used to determine the bivariate patient-specific thresholds which split the data into regions with different strength of dependence between nurses' rating and the automated sedation dose, namely: (1) occurrences with moderate agitation (the main region), (2) occurrences with mild agitation (the lower tail region), and (3) occurrences with severe agitation (the upper tail region). The K-plot (Genest and Boies (2003)) adopts the familiar probability plot (Q-Q plot) to detect dependence. In the absence of association between two variables, the K-plot is close to a straight line, while the amount of curvature in the K-plot is characteristic of the degree of dependence in the data, and is related, in a definite way, to the underlying copula. This method is closely related to Kendall's tau-statistic (Kendall (1938)), from which it takes the name.

The construction of a K-plot requires ordering $H_{i}$, as $H_{(1)} \leq \ldots \leq H_{(n)}$. For a given pair $\left(X_{i}, Y_{i}\right)$ with $1 \leq i \leq n, H_{i}$ is defined as follows:

$$
H_{i}=\frac{1}{n-1} \#\left\{j \neq i: X_{j} \leq X_{i}, Y_{j} \leq Y_{i}\right\}
$$

Now, using the definition of the density of an order statistic, we have the expected value $W_{(i: n)}$ for the $i^{t h}$ order statistic $H_{(i)}$ under the null hypothesis of independence for all $1 \leq i \leq n$ :

$$
W_{i: n}=n\left(\begin{array}{c}
n-1 \\
i-1
\end{array}\right) \int_{0}^{1} \omega K_{0}(\omega)^{i-1} \times\left\{1-K_{0}(\omega)\right\}^{n-1} d K_{0}(\omega) .
$$

Now to obtain a K-plot, we plot the pairs $\left(W_{i: n}, H_{(i)}\right)$, for $1 \leq i \leq n$ where $W_{i: n}$ is the expectation of the $i^{\text {th }}$-order statistic in a random sample of size $\mathrm{n}$, drawn from the distribution $K_{0}$ of the $H_{i}$, under the null of independence. The form of the bivariate distribution $K_{0}$ is given as follows:

$$
K(\omega)=K_{0}(\omega)=\operatorname{Pr}\{U V \leq \omega\}=\omega-\omega \log (\omega), \quad 0 \leq \omega \leq 1
$$

where $\mathrm{U}$ and $\mathrm{V}$ are independent uniform random variables on the interval [0,1]. This choice of $K_{0}(\omega)$ is then used to compute the $W_{i: n}$ required for the plot.

To detect patient-specific thresholds, first, we estimate the best fitting copula using maximum likelihood estimation (Brechmann (2010)) which informs us about the presence of tails. Then, we compute the sum of squared residuals (SSR) of the relevant segments for the dependence measure $H_{i}$ from K-plot. Next, we employ the dynamic programming algorithm (Bai and Perron (2003)) to estimate tail thresholds by minimising SSRs.

\section{RESULTS}

In this section, we consider two patients from the pool of 37 patients. All patients were classified into poor and good trackers based on the Wavelet Probability Bands (WPB) created using Bayesian wavelet shrinkage methods (Kang et al. (2011) and Kang et al. (2013)). A good tracker is a patient whose simulated profiles was close to the mean profile, a poor tracker is a patient for whom this was not the case (Kang et al. (2011)). While patient 28 has a WPB of $50.8 \%$ and is considered a poor tracker, patient 18 has a WPB of $93.8 \%$ and is considered a good tracker.

The line plots of nurses' rating of a patient's agitation and the automated sedation dose shown in Fig.1 (a) and (b) indicate that two variables tend to move together. The scatter plots in Fig.1 (c) and (d) show that most of the observations are close to the regression line with some observations lying away from the main cluster of the data. As such, a few of the nurses' ratings for patient 18 are unusually high. Moreover, the variance of nurses' ratings is increasing with the magnitude of the drug infusion dose. The best-fitting copula for both patients is the Survival BB8. Two goodness-of-fit tests (the p-values for both Cramer-von Mises and KolmogorovSmirnov tests are $<0.05)$ support the copula fit for both patients. This copula has the tail dependence when the parameter $\delta=1$ (Joe (1997)). For both patients, this parameter is close to one indicating that both joint distributions have tails. Using $H_{i}$ measures from K-plot we identify tail thresholds for both patients that are shown as dashed vertical lines on Fig.1 (e) and (f). Fig.2. (a) and (b) show histograms with tail thresholds for both patients. It is obvious from histograms that the range of nurses' ratings is greater for patient 18. Table 1 provides the details on the tail thresholds for both patients. Note that the lower threshold for nurses' rating is lower for the poor tracker, whereas the upper threshold is higher for the good tracker compared with the poor tracker.

All, except one (the coefficeint for the main region in equation (4), where p-value is 0.12 ) of the estimated regression coefficients in equations (4) and (5) are statistically significant with p-values $<0.05$.

$$
\text { Score }=0.62 \text { Dose }+0.83(\text { Main region }==" Y e s ")+6.02(\text { Upper tail }==" Y e s ")
$$


A. Tursunalieva et al., Copula modelling of agitation-sedation rating ...

\begin{tabular}{llllll}
\hline $\begin{array}{l}\text { Type of } \\
\text { tracker }\end{array}$ & Sample & \multicolumn{2}{c}{ Order statistic } & \multicolumn{2}{c}{ Nurses' rating } \\
size & Lower tail & Upper tail & Lower threshold & Upper threshold \\
\hline Good (18) & 63 & 15 & 51 & 1.3 & 6.1 \\
Poor (28) & 203 & 50 & 153 & 1.1 & 2.6 \\
\hline
\end{tabular}

Table 1. Threshold details for both patients.

For patient 18, an estimated slope coefficient indicates that on average for an additional $\mathrm{mg} / \mathrm{ml}$ of drug infusion dose nurses' rating of the patient's agitation severity increases by 0.62 points. The mean nurses' rating when a patient is expressing signs of a moderate agitation (the main region) is approximately the same as the mean nurses' rating when a patient is expressing signs of a mild agitation (since the estimated coefficient is statistically not different from zero). The mean nurses' rating when a patient is expressing signs of a severe agitation (the upper tail region) is 6.01 points higher than the mean nurses' rating when a patient is experiencing a mild or moderate agitation.

$$
\text { Score }=0.48 \text { Dose }+0.91(\text { Main region }==" Y e s ")+2.52(\text { Upper tail }==" Y e s ")
$$

For patient 28, an estimated slope coefficient indicates that on average for an additional $\mathrm{mg} / \mathrm{ml}$ of drug infusion dose nurses' rating of the patient's agitation severity increases by 0.48 points. The mean nurses' rating when a patient is expressing signs of a moderate agitation (the main region) is 0.91 points higher than the mean nurses' rating when a patient is expressing signs of a mild agitation. The mean nurses' rating when a patient is experiencing a severe agitation (the upper tail region) is 2.52 points higher than the mean nurses' rating when a patient is expressing signs of a mild agitation.

The strength of the linear relationship between nurses' rating of a patient's agitation level and the automated sedation dose is stronger for the good patient (the slope coefficient is equal to 0.62), compared with the poor patient (the slope coefficient is equal to 0.48). Incorporating the upper and lower tail dummy variables improved predictions of the nurses' rating by increasing the adjusted $R^{2}$ values from 0.58 and 0.56 for the simple linear regression to 0.86 and 0.84 for the regression with tail dummy variables for patients 18 and 28 respectively.

Kendall's correlation test is used to test the null hypothesis that $\tau=0$. The alternative hypothesis is that the variables are correlated, and $\tau$ is non-zero. The results are reported in Table 2, where $\tau$ value with an asterisk indicates a statistically significant value. The lower tail dependence is the strong and significant for both patients, indicating that when a patient is expressing signs of a mild agitation, the correlation between nurses rating and drug infusion is the strongest. The dependence in the main region is weaker compared with the lower tail dependence, but stronger than the upper tail dependence. It is only significant for the good tacker (Patient 18). The shapse of the concentration ellipses on the scatterplots in Fig.2 (c) and (d) indicate that the dependence between the nurses' rating and the automated infusion dose is strong in the lower tail and main regions for a good tracker and only in the lower tail region for a poor tracker.

The last three columns of Table 2 provide the percentage of observations from the copula regions that are common in the respective WPBs. For example, we count the number of observations that are common to both the lower tail and the lower WPB (where nurses' rating is lower than lower WPB). The percentage of lower and upper tail observations that are common with the lower and upper WPBs is higher for the poor tracker (11\% vs $48.4 \%$ and $82 \%$ vs $96.2 \%$ respectively for the lower and upper tail) than the good tracker. However, the percentage of observations that are common to both the main region (the region associated with moderate agitation intensity) and within WPB is higher for the good tracker (74.0\%) compared to the poor tracker $(52.2 \%)$.

Table 2. Kendall's correlations and percentage of observations common to copula regions and WPBs.

\begin{tabular}{lllllll}
\hline $\begin{array}{l}\text { Type of } \\
\text { tracker }\end{array}$ & Lower WPB & $\begin{array}{c}\text { Kendall's } \tau \\
\text { Within WPB }\end{array}$ & Upper WPB & $\begin{array}{l}\text { Lower tail } \\
\text { Lower WPB }\end{array}$ & $\begin{array}{l}\text { Main region } \\
\text { Within WPB }\end{array}$ & $\begin{array}{l}\text { Upper tail } \\
\text { Upper WPB }\end{array}$ \\
\hline Good (18) & $0.65^{*}$ & $0.57^{*}$ & 0.38 & 11.0 & 74.0 & 82.0 \\
Poor (28) & $0.71^{*}$ & 0.30 & 0.23 & 48.4 & 52.2 & 96.2 \\
\hline
\end{tabular}


A. Tursunalieva et al., Copula modelling of agitation-sedation rating ...

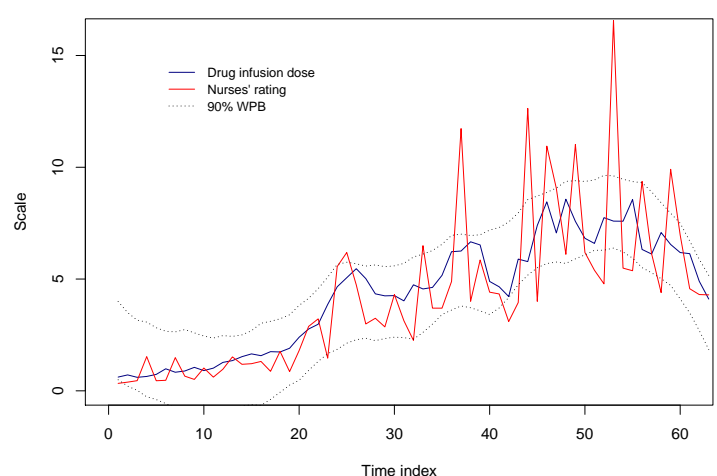

(a) Line plot with WPBs for patient 18 .

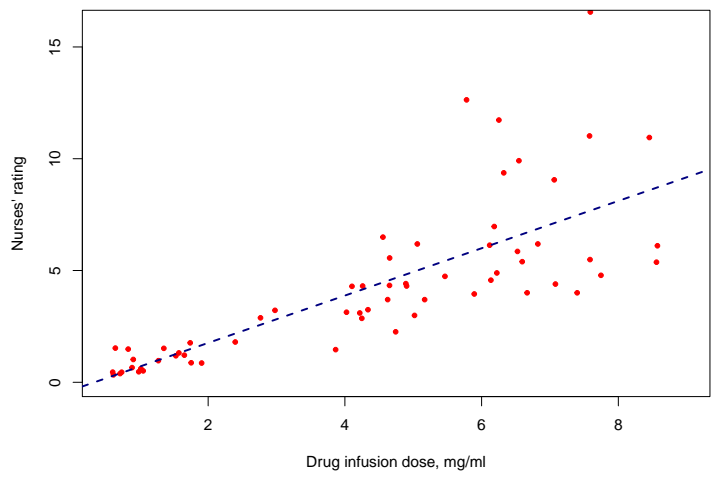

(c) Scatter plot with the line of the best fit for patient 18 .

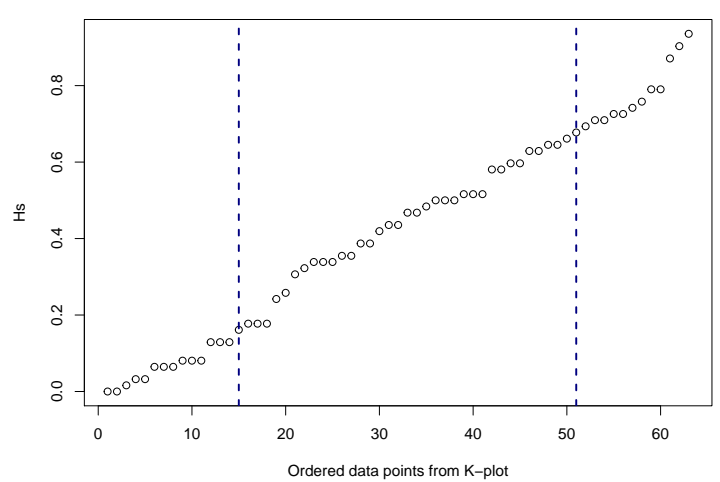

(e) $H_{i}$ measure plot with the bivariate threshold (dashed line) for patient 18 .

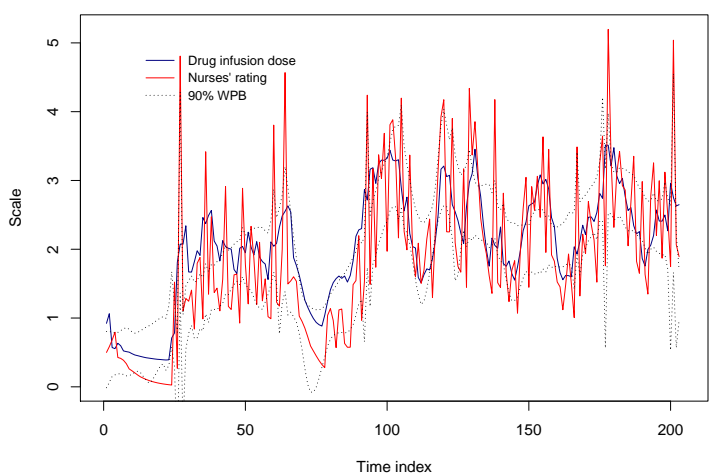

(b) Line plot with WPBs for patient 28.

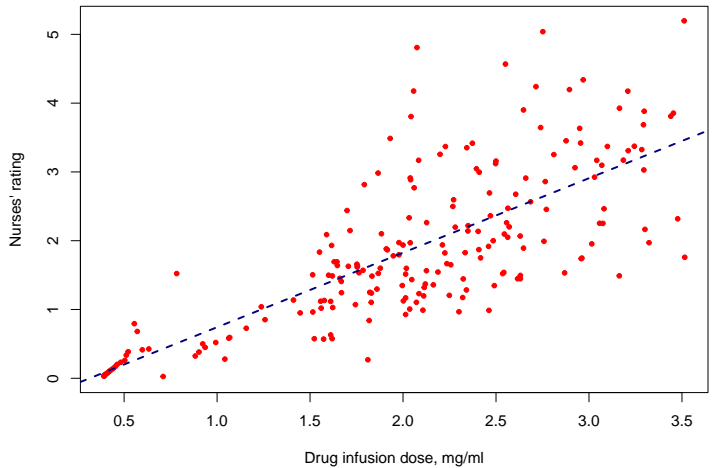

(d) Scatter plot with the line of the best fit for patient 28 .

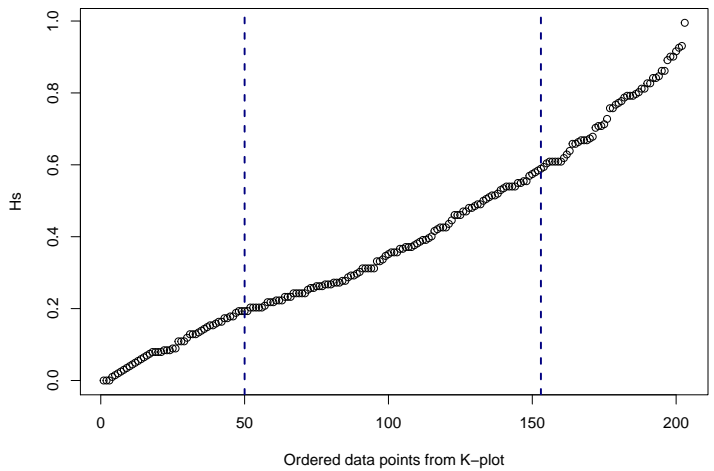

(f) $H_{i}$ measure plot with the bivariate threshold (dashed line) for patient 28.

Figure 1. Line plots, scatterplots, and dependence plots

\section{DISCUSSION AND CONCLUSION}

The paper aimed to integrate the non-elliptical dependency structure between nurses' rating of a patient's agitation level and the automated sedation dose to account for nonlinear relationships between the two variables. Using copula-based dependence measure to identify lower and upper tail threshold allows us to capture the relationship between nurses' rating of a patient's agitation level and the automated sedation dose in different 


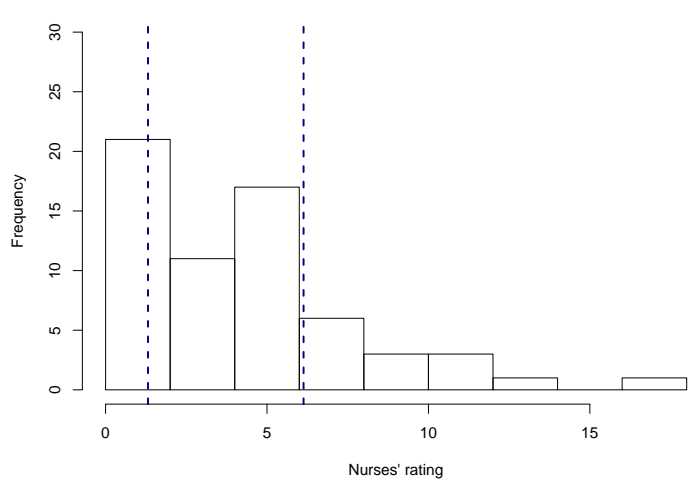

(a) Histogram for the nurses' rating with tail thresholds (dashed lines) for patient 18.

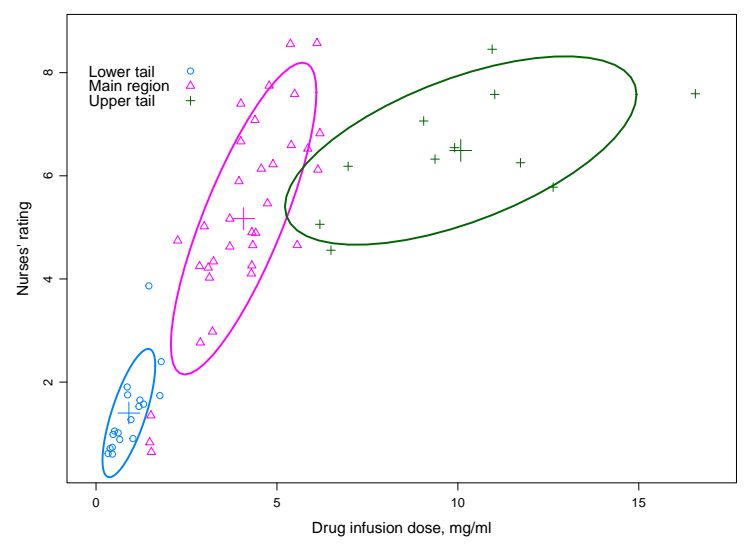

(c) Scatterplot with regions for patient 18 .

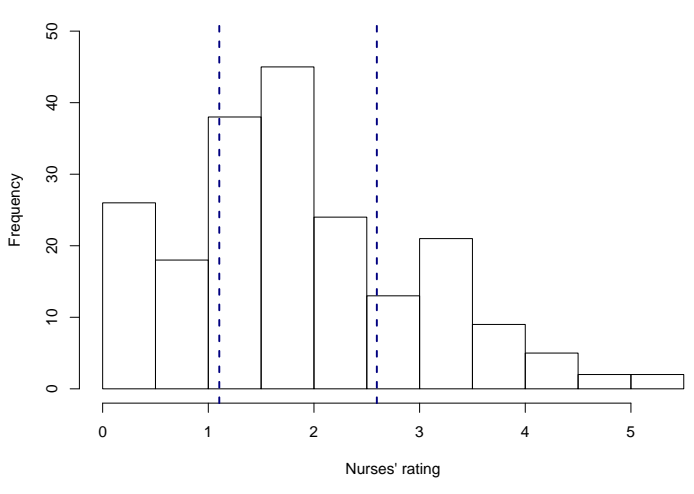

(b) Histogram for the nurses' rating with tail thresholds (dashed lines) for patient 28.

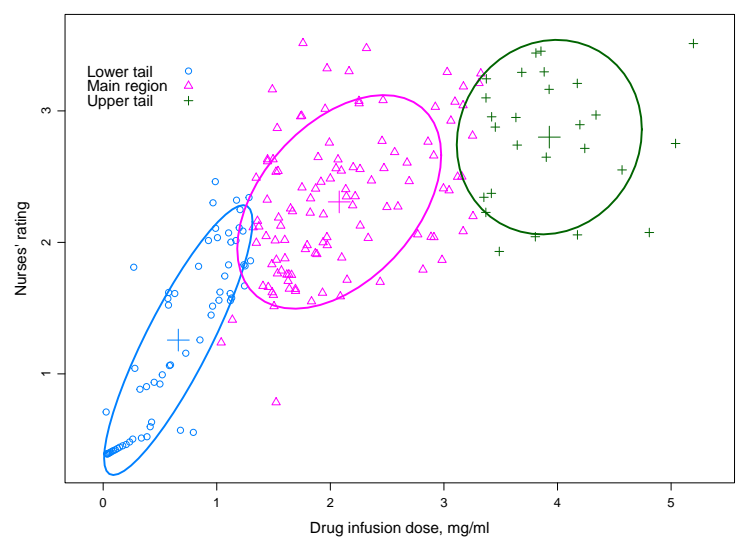

(d) Scatterplot with regions for patient 28 .

Figure 2. Histograms with thresholds, the scatterplots with regions, and mosaic plots.

agitation severity regions. The estimated copulas provide valuable information regarding whether tail dummy variables need to be included in the regression model. The best-fitting c opula shows that the dependency structure between the nurses rating of a patients agitation level and the administered dose of sedative for both patients has an upper and lower tail. More specifically, a correlation between the nurses rating of a patients agitation level and the administered dose of the sedative is the strongest when patients are experiencing mild agitation and weakest when patients are experiencing severe agitation. The results show that for a good tracker, the nurses' rating of the patients' agitation levels has strong positive correlation with the administered dose of the sedative for low and mild agitation severity. For a poor tracker, the nurses' rating of the patients' agitation levels has strong positive correlation with the administered dose of the sedative only for low agitation severity.

Incorporating the tail dummy variables improved predictions of the nurses' rating by increasing the adjusted $R^{2}$ values by $28 \%$. Moreover, the strongest degree of association between the nurses rating within WPB and observations from the region regions is observed when a patient is experiencing severe agitation intensity.

In this paper we have accounted for nonlinear relationships between the two variables, finding thresholds and regions of mismatch between the nurse's scores and sedation dose, thereby suggesting a possible way forward for the improved alerting system for over/under-sedation. Better predictions and quality of care will have consequent policy implications for improving hospital performance by achieving better health outcomes, improved access to health services, and less waste for the same level of healthcare costs. The copula approach suggested in this paper is generalisable to any study which investigates the similarity or closeness of bivariate time series of, for example, a large number of units (patients, households) and of time series of disparate lengths and possibly long length. The aim of future work on the full set of 37 patients is to generalise the 
A. Tursunalieva et al., Copula modelling of agitation-sedation rating ...

results and show that the copula approach can accurately capture the dynamic (temporal) dependence between the nurses' rating, and the automated infusion dose and map the copula regions with the respective WPBs. Further developments could include investigation of commonality of dependence strengths, best-fit copulas, and complexity of a dependence structure for good and poor trackers to use the most effective treatment plans.

\section{REFERENCES}

Bai, J. and P. Perron (2003). Computation and analysis of multiple structural change models. Journal of Applied Econometrics 18(1), 1-22.

Barr, J., G. L. Fraser, K. Puntillo, G. C. Ely, E. W. and, J. F. Dasta, J. E. Davidson, J. W. Devlin, J. P. Kress, A. M. Joffe, D. B. Coursin, D. L. Herr, A. Tung, B. R. Robinson, D. K. Fontaine, M. A. Ramsay, R. R. Riker, C. N. Sessler, B. Pun, Y. Skrobik, and R. Jaeschke (2013). Clinical practice guidelines for the management of pain, agitation, and delirium in adult patients in the intensive care unit. Critical Care Medicine 41(1), 263-306.

Boero, G., P. Silvapulle, and A. Tursunalieva (2010). Modelling the bivariate dependence structure of exchange rates before and after the introduction of the euro: a semiparametric approach. International Journal of Finance and Economics 16(4), 357-374.

Brechmann, E. C. (2010). Truncated and simplified regular vines and their applications. Diploma thesis, Technische Universitaet Muenchen.

Chase, J. G., A. D. Rudge, G. M. Shaw, G. C. Wake, D. Lee, and I. L. Hudson (2004). Modeling and control of the agitation-sedation cycle for critical care patients. Medical Engineering and Physics 26(6), 459-471.

Embrechts, P.and Lindskog, F. and A. McNeil (2003). Modelling dependence with copulas and applications to risk management (Handbook of Heavy Tailed Distributions in Finance ed.). Elsevier.

Fraser, G. L. and R. R. Riker (2001). Monitoring sedation, agitation, analgesia, and delirium in critically ill adult patients. Critical Care Clinics 17(4), 967-987.

Genest, C. and J.-C. Boies (2003). Detecting dependence with kendall plots. The American Statistician 57(4), 275-284.

Joe, H. (1997). Dependence Modeling with Copulas. Chapman and Hall.

Kang, I., I. L. Hudson, A. Rudge, and J. G. Chase (2011). Discrete Wavelet Transforms, Chapter Wavelet signatures and diagnostics for the assessment of ICU Agitation-Sedation protocols., pp. 321-348. InTechOpen.

Kang, I., I. L. Hudson, A. Rudge, and J. G. Chase (2013). A Compendium of New Approaches and Recent Applications, Chapter Density estimation and wavelet thresholding via Bayesian methods: A Wavelet Probability Band and related metrics to assess agitation and sedation in ICU patients., pp. 127-162. InTechOpen.

Kendall, M. (1938). A new measure of rank correlation. Biometrika 30(1/2), 81-93.

Kress, J. P., A. S. Pohlman, and J. B. Hall (2002). Sedation and analgesia in the intensive care unit. American Journal of Respiratory and Critical Care Medicine 166(8), 1024-1028.

Milane, T. A.; Bennett, E. D. . G. R. M. (1992). Isoflurane and propofol for long-term sedation in the intensive care unit. a crossover study. Anaesthesia 47, 768-774.

Nelsen, R. (2006). An Introduction to Copulas. Springer Science+Business Media Inc., New York, NY 10013, USA.

Rudge, A. D., J. G. Chase, G. M. Shaw, and D. Lee (2006a). Physiological modelling of agitation-sedation dynamics. Medical Engineering and Physics 28(1), 49-59.

Rudge, A. D., J. G. Chase, G. M. Shaw, and D. Lee (2006b). Physiological modelling of agitation-sedation dynamics including endogenous agitation reduction. Medical Engineering Physics 28(7), 629-638.

Rudge, A. D., J. G. Chase, G. M. Shaw, D. Lee, G. C. Wake, and I. L. Hudson (2005). Impact of control on agitation-sedation dynamics. Control Engineering Practice 13(9), 1139-1149.

Sessler, C. N., M. S. Gosnell, M. J. Grap, G. M. Brophy, P. V. O’Neal, and K. A. Keane (2002). The Richmond agitation-sedation scale: validity and reliability in adult intensive care unit patients. American Journal of Respiratory and Critical Care Medicine 166(10), 1338-1344.

Tursunalieva, A., I. Hudson, and G. Chase (2018). Improved prediction of a nurse's agitation-sedation rating in relation to automated sedation infusion levels in intensive care: a copula approach. In Joint International Society for Clinical Biostatistics and Australian Statistical Conference, Melbourne, Australia.

Tursunalieva, A., I. Hudson, and G. Chase (2019). Copula modelling of nurses' agitation-sedation rating of ICU patients. In Springer Communications in Computer and Information Science (forthcoming).

Vincent, J., Y. Shehabi, T. S. Walsh, P. P. Pandharipande, J. A. Ball, P. Spronk, D. Longrois, T. Strm, G. Conti, G. Funk, R. Badenes, J. Mantz, C. Spies, and J. Takala (2016). Comfort and patient-centred care without excessive sedation: the ecash concept. Intensive Care Medicine. 